\title{
CIUDADANÍA PARTICIPATIVA Y TRANSFORMADORA: ANÁLISIS DE DISCURSOS Y PROPUESTAS DE APRENDIZAJE
}

\author{
Participative and transformational citizenship: analysis \\ of discourses and learning proposals
}

\section{Citoyenneté participative et pour la transformation: analyses des discours et propositions d'apprentissage}

\author{
Patricia Mata-Benito*, Belén Ballesteros-VelázQuez* y María Teresa Padilla- \\ CARMONA $^{\text {*** }}$ \\ * Universidad Nacional de Educación a Distancia. Facultad de Educación. \\ C/ Juan del Rosal, 14. 28040 Madrid. Correo-e: pmata@edu.uned.es; bballesteros@ \\ edu.uned.es \\ ** Universidad de Sevilla. Facultad de Ciencias de la Educación. C/ Pirotecnia, \\ s/n. 41013 Sevilla. Correo-e: tpadilla@us.es
}

Fecha de recepción: marzo de 2013

Fecha de aceptación definitiva: junio de 2013

Biblid [(1130-3743) 25, 2-2013, 49-68]

RESUMEN

Se parte de considerar la ciudadanía como un concepto dinámico y relacional: una práctica orientada al desarrollo de capacidades y poderes colectivos para la creatividad, la acción y la transformación social. Desde esta perspectiva, realizamos un estudio basado en entrevistas en profundidad a 14 informantes, cuya finalidad es explorar los discursos y procesos a través de los que se define y pone en juego la ciudadanía. El análisis del contenido de las entrevistas nos permite profundizar en sus opiniones y experiencias en torno a los significados y el aprendizaje de la ciudadanía. Los resultados que presentamos en este trabajo se centran en este último 
aspecto: cómo se aprende, a través de qué referentes, cuáles son las motivaciones para actuar y cómo se educa. Asimismo, se ofrecen algunas propuestas tendentes a facilitar una educación ciudadana comprometida con el cambio social.

Palabras clave: ciudadanía participativa, ciudadanía como práctica, transformación social, aprendizaje de la ciudadanía, educación para la ciudadanía, entrevistas en profundidad.

\section{SUMMARY}

Our article deals with citizenship as a dynamic and relational concept: as a practice that points to the development of collective skills and powers to promote creativity, action and social transformation. From this approach, we have carried out a research based on in-depth interviews to 14 participants, with the aim of exploring the discourses and processes by means of which citizenship is defined and put into action. Our analysis deepens in the participants opinions about citizenship meanings and learning. The results showed in this paper focus on how it is learnt, by means of which referents, which the motivations are, and how it is trained. Likewise, we offer some proposals to promote a citizenship education aiming to social change.

Key words: participative citizenship, citizenship as a practice, social transformation, citizenship learning, citizenship education, in-depth interviews.

\section{SOMMAIRE}

Dans cet article on propose d'abord la considération de la citoyenneté comme un concept dynamique et relationnel: une pratique orientée vers le développement des capacités et pouvoirs collectifs pour la créativité, l'action et la transformation sociale. De ce point de vue on a développé un étude que s'appuie sur des entretiens approfondis avec 14 informateurs. Le but de ces entretiens est l'exploration des discours et des processus par lesquels la citoyenneté est définie et mise en jeu. L'analyse du contenu des entretiens nous donne un aperçu de leurs points de vue et expériences sur l'apprentissage et les significations de la citoyenneté. Les résultats présentés dans ce document vont souligner certains aspects: comment on apprend, quelles sont les références, quelles sont les motivations, comment a été le processus éducatif. Il offre aussi des propositions destinées à faciliter éducation à la citoyenneté engagée au changement social.

Mots clés: citoyenneté participative, citoyenneté en tant que pratique, transformation sociale, apprentissage de la citoyenneté, éducation à la citoyenneté, entretiens approfondis. 


\section{INTRODUCCIÓN}

La perspectiva de la equidad en educación pone de manifiesto las tensiones existentes entre diversidad e igualdad, entre reconocimiento y redistribución. Nos enfrentamos a no pocas paradojas: mientras el vínculo entre diferencia y desigualdad se consolida como una constante, los resultados de la investigación (Aguado, 2006, 2010; Malik y Mata, 2009) señalan que la diversidad se asocia persistentemente a carencia o problema, mientras que la educación intercultural se reduce a la atención educativa a grupos específicos, etiquetados y clasificados como diferentes, y generalmente considerados como deficitarios respecto a un determinado estándar de normalidad. Como consecuencia, determinadas personas y grupos ven sistemáticamente reducidas sus oportunidades educativas y vitales, y limitada su participación social.

Algunas de las razones que subyacen a esta situación paradójica apuntan al dominio de la perspectiva diferencial-culturalista en el ámbito educativo. Esta perspectiva se asienta, por un lado, en una visión esencialista de las culturas y las identidades, cuya consecuencia es la categorización y clasificación de personas y grupos. Por otro, en la fácil acomodación que encuentran estas categorías en los sistemas educativos, que pueden utilizarlas como criterio para simplificar y organizar sus prácticas, al tiempo que como justificación de su incapacidad para desafiar el proceso de reproducción de las desigualdades (Mata y Ballesteros, 2012).

Sin embargo, las identidades y las culturas se encuentran en estado de construcción e interacción permanente; de ahí la imposibilidad de definir a los otros al margen de nuestra relación con ellos (Abdallah-Pretceille, 2001). Culturas e identidades se "imaginan" y se construyen en contextos concretos, definidos por relaciones políticas, económicas y sociales determinadas por el ejercicio desigual del poder (Dietz, 2003). El enfoque intercultural se propone como un discursopráctica que aborda la diversidad y la desigualdad, el pluralismo y la complejidad. Propone un proceso de aprendizaje lento y constante, basado en la reflexión, la toma de conciencia y la revisión crítica de nuestras ideas y de nuestras prácticas (Martinelli, 2000), y orientado a la construcción de ciudadanía. Esto implica, por una parte, la concienciación y el posicionamiento contra la desigualdad y la exclusión, frente a la dominación, la explotación y sus mecanismos de apoyo. Por otra, el fortalecimiento de las personas como actores sociales y sujetos culturales, y el desarrollo de las capacidades y poderes colectivos para la creatividad, la acción y la transformación social. Es decir, la construcción de una ciudadanía crítica, participativa y transformadora.

La ciudadanía, una categoría política que alude a la pertenencia y la participación de las personas en la sociedad, ha adquirido relevancia social y educativa en los últimos años. Diversos autores (Benedicto y Morán, 2003; Schugurensky, 2005; Schugurensky y Myers, 2008) inciden en la necesidad de investigar en profundidad acerca de cómo se construye la ciudadanía en los procesos educativos, entendidos 
en su dimensión más amplia, como procesos de aprendizaje continuo a lo largo de toda la vida. Turner $(1990,1993)$ apunta asimismo la necesidad de investigar sobre la ciudadanía a partir de la observación y descripción de los contextos de experiencia y de actividad de las personas en su vida cotidiana. Por su parte, Siim (2000) insiste en que la comprensión del concepto de ciudadanía, más allá de ideales normativos y discursos del deber ser, pasa por la investigación empírica sobre cómo opera el principio de ciudadanía en la vida social y política, cuáles son sus características en grupos humanos concretos y cómo los individuos acceden a la condición de ciudadanos.

El interés por la comprensión del concepto de ciudadanía orienta este trabajo en el que presentamos el proceso y los resultados de una investigación que ha explorado cómo se entiende, cómo se ejerce y cómo se aprende la ciudadanía a través de un estudio cualitativo basado en entrevistas en profundidad.

\section{De la CIUdAdANía COMO estatus a la CiUdAdanía COMO PRÁCTiCA}

La idea de la ciudadanía como factor de igualdad social es elaborada por primera vez por Marshall (1998) quien, en 1950, desarrolla el concepto de ciudadanía como estatus, asociado a la pertenencia como miembro de pleno derecho a un Estadonación. Se plantea la hipótesis de que la combinación de derechos civiles, políticos y sociales, asociada al reconocimiento del estatus de ciudadano, acabaría conduciendo hacia la igualdad. Diversos autores (Heater, 2007; Turner, 2001) señalan la inconsistencia de esta hipótesis: por una parte, porque la ciudadanía como estatus implica de hecho la construcción de identidades basadas en asunciones acerca de la etnicidad, la religión e incluso la sexualidad, constituyéndose sobre las diferencias y excluyendo el acceso de "los otros" a los recursos en base a su identidad de género, étnica o nacional. Por otra, porque esta ciudadanía no es suficiente para garantizar los derechos efectivos, ya que no da cuenta de los procesos capaces de explicar el avance de los mismos. Por último, porque la falta de realización de estos la convierte en un título o identidad formal vacía de significado.

La ciudadanía estatus conecta con un determinado "modelo" de ciudadano que se define en función de criterios de homogeneidad y que, en el mejor de los casos, se caracteriza por la pasividad y la tutela. A partir de los años ochenta, con la caída del modelo estatal y el predominio del mercado, las teorías económicas neoliberales irrumpen en la política, lo que conlleva un cambio importante en la construcción de sentidos e identidades sociales, transformando al ciudadano en consumidor. Los modelos de ciudadanía resultantes son el de ciudadano cliente en el ámbito social, consumidor a nivel económico y elector en política. Estos modelos actúan en el marco de la elección individual sobre productos o propuestas preestablecidas y ofertadas. La esfera pública, el espacio de lo común, se desdibuja, y con ello se afianza la apatía y el desinterés político, planteando nuevos retos a la reconstrucción de la ciudadanía (Capella, 2005; Rius, 2010; Turner, 2001). 
En este contexto se hace evidente que el modelo de ciudadanía como asignación de derechos dentro de un Estado-nación basado en la ilusión de realidades homogéneas, está agotado. Es urgente incorporar la gramática de la diversidad a la organización moral y política (Susín, 2008). En este sentido, el reto de revertir la tendencia a la erosión de la ciudadanía pasa por la creación y multiplicación de alternativas social y ambientalmente sostenibles en comunidades a pequeña escala (Ikeda, 2004).

Estas propuestas y alternativas se articulan en un fenómeno que Moro (2008) denomina "nueva ciudadanía". Mientras las formas tradicionales de ciudadanía política (el voto, la pertenencia a partidos políticos, sindicatos, etc.) se debilitan, aumenta la exigencia y la práctica ciudadana de la participación democrática. A través de grupos diversos -asociaciones, movimientos, comunidades, organizaciones de todo tipo, iniciativas monotemáticas, redes, etc.- y en relación con un amplio abanico de cuestiones -medio ambiente, exclusión, cultura, educación, salud, desarrollo, cooperación-, la nueva ciudadanía pretende participar en la elaboración de las políticas públicas. El significado de la ciudadanía se vincula así a la práctica. Se concibe como un concepto dinámico y relacional, una forma colectiva de pertenencia activa a la comunidad que implica el desarrollo de identidades y sentimientos de solidaridad e implicación (Benedicto y Morán, 2003).

\section{El Discurso EDUCATIVO: EDUCACión CÍviCA VERSUS APRENDIZAJE DE LA CIUDADANÍA}

Si entendemos la ciudadanía no como estatus, sino como una práctica que debe aprenderse, la educación ciudadana debería configurarse como una educación crítica y participativa. Sin embargo, una revisión de los discursos educativos en torno a la ciudadanía nos coloca ante un escenario en el que predomina un enfoque teórico-normativo desde el cual se dictan modelos que definen a priori qué es ser buen ciudadano. A partir de estos modelos se elaboran los programas de educación "para" la ciudadanía, concebida mayoritariamente como una educación cívica con escasos componentes críticos.

La educación cívica aspira a complementar los derechos de la ciudadanía con las virtudes que configuran al "buen ciudadano" (Mougán, 2009). Estas propuestas se configuran sobre la base del civismo, entendido como un comportamiento ético-político, junto a la consideración de la educación como una "preparación para" el ejercicio futuro de la ciudadanía (Da Silveira, 2009), un proceso "capaz de transformar al ciudadano pasivo y conformista en un ciudadano activo y exigente" (Rubio Carracedo, 2003, 211). Podemos encontrar un ejemplo de este discurso en el objetivo que se plantea la asignatura «Educación para la Ciudadanía y los Derechos Humanos" en nuestro país (hasta hoy; el proyecto de la LOMCE prescinde de esta en el mapa curricular de la enseñanza obligatoria, proponiéndose en su lugar una materia con una orientación cívica y constitucional). Según el Real Decreto por el que se establecen las enseñanzas mínimas correspondientes a la Educación 
Secundaria Obligatoria, "la Educación para la Ciudadanía tiene como objetivo [...] la formación de futuros ciudadanos con criterio propio, respetuosos, participativos y solidarios, que conozcan sus derechos, asuman sus deberes y desarrollen hábitos cívicos para que puedan ejercer la ciudadanía de forma eficaz y responsable» (MEC, 2006b, 715).

DeJaeghere (2009) describe las características de la educación para la ciudadanía en diversos países que, en su análisis, se desarrolla en un continuo entre mínimos y máximos. El enfoque cívico se situaría cerca de los mínimos, incluyendo:

ideas normativas de la ciudadanía, tales como la atribución jurídica de una identidad ciudadana, y los derechos y deberes cívicos [...] enfatiza en los derechos y la igualdad de todos en una democracia, pero no tiene en cuenta la diversidad de experiencias [...] tienden a centrarse en los contenidos, privilegiando los conocimientos cívicos y prestándole poca atención a los procesos y la participación ciudadana. Su objetivo es promover un "buen" ciudadano, que sea respetuoso con la ley, contribuya a la sociedad y posea un buen carácter [...] no se ocupa de las estructuras sociales y las relaciones que crean desigualdades entre los ciudadanos (DeJaeghere, 2009, 228).

Desde otra perspectiva, Seoane (2003) presenta una propuesta de educación "desde" la ciudadanía, que explora el contexto relacional en el que se desenvuelve su aprendizaje. Esta educación se distancia de la definición de un modelo prefijado en torno al "buen ciudadano", entendiendo la ciudadanía como un modo de relacionarse con los otros basado en vínculos previos con ellos. No existe el individuo como ser que se sitúa "frente a" la realidad y los otros, y toma decisiones por cuenta propia contando únicamente con su razón. Los vínculos sociales, argumenta Seoane, son previos a la reflexión moral, que se produce "desde" la ciudad y "con" los otros.

\section{Propuestas EDUCATIVAS PARA LA CONSTRUCCIÓN DE CIUDADANÍA}

Estudios recientes tratan de sistematizar las experiencias para el aprendizaje de la ciudadanía promovidas desde diferentes instancias educativas. Desde nuestro punto de vista, no resulta una tarea fácil por distintos motivos. De una parte, el propio uso del término ciudadanía conduce a identificar prácticas que responden a enfoques desiguales, sin que en todas ellas podamos hablar de una participación orientada a la transformación social. Por otra parte, la referencia a "aprendizaje" puede llevar a establecer una línea de separación artificial entre espacios de ejercicio y espacios de aprendizaje. En este sentido, los presupuestos participativos, por ejemplo, concebidos como posibilidad para la intervención de la ciudadanía en la toma de decisiones municipales (Folgueiras, 2005), no suelen identificarse como ámbitos de aprendizaje de la ciudadanía (Argibay, Celorio, Celorio y López de Munain, 2011). 
Los espacios que se vinculan al aprendizaje de la ciudadanía presentan una heterogeneidad de experiencias, tanto en el ámbito de la educación formal como no formal. De entre ellos, destacamos los siguientes:

- $\quad$ Las comunidades de aprendizaje. Desde esta práctica, se parte del diálogo basado en la igualdad entre todos los agentes educativos con la intención de dar voz a toda la diversidad de personas de una comunidad. Así, la entrada en la escuela de personas con perfiles muy heterogéneos produce transformaciones no solo en los niveles de aprendizaje, sino también en el contexto social y cultural (Flecha, 2009). Este enfoque se plantea además la necesidad de cambiar las relaciones, pasando de relaciones de poder a relaciones dialógicas.

- Red de Escuelas Democráticas. Apuestan por un cambio educativo promovido por los propios protagonistas cuyo fin es educar en una ciudadanía activa y transformadora. Actúan desde los principios de equidad, inclusión, cooperación, apertura a la comunidad, participación y justicia social (López Ruiz, 2008).

- Los Planes Educativos de Entorno. Se pretende diseñar de forma participativa una acción educativa abierta a todos los alumnos, especialmente a aquellos que pertenecen a sectores sociales vulnerables, para fomentar el éxito, la convivencia, la integración cultural y las expectativas sociales (Argibay et al., 2011).

- $\quad$ Red de aprendizaje-servicio. Promueve actividades que combinan el servicio a la comunidad con el aprendizaje de conocimientos, habilidades y valores que permitan la formación de competencias reflexivas y críticas para el ejercicio responsable de la ciudadanía (Puig, Gijón, Martín García y Rubio, 2011).

- La Educación Popular. Concibe la participación como condición y resultado de un desarrollo social que se enfrenta a la pobreza y a la injusticia. Se fomenta la producción colectiva de conocimiento, aunando práctica y teoría para la construcción de un proyecto político-social que luche contra las desigualdades y discriminaciones de todo tipo (Mata, 2010).

- Las Ciudades Educadoras. Buscan romper la rigidez del sistema educativo integrando, a través del diálogo y la cooperación, la educación formal, no formal e informal (Folgueiras, 2005).

- Los Consejos Infantiles. Son órganos municipales de participación que reúnen a niños y niñas para reflexionar, debatir y hacer propuestas sobre aspectos de la ciudad que pueden mejorar sus vidas y las de todos los ciudadanos en general (Trilla y Novella, 2011).

Lo común a estas experiencias se encuentra en la búsqueda del diálogo y la participación como condiciones básicas para la convivencia social y por tanto para la educación, cuyo objetivo precisamente es crear situaciones en las que se dé este diálogo intersubjetivo, en un contexto democrático y de igualdad (Molina, 2000). Sin embargo, se nos plantean algunos interrogantes: 
- ¿Responden a un modelo educativo global? Algunas experiencias que se plantean como tales, como los Planes Educativos de Entorno, se concretan en la práctica como un modelo fragmentado. Como señala Bargalló (2008), estos se ubican casi exclusivamente en el espacio extraescolar, con escasa permeabilidad entre el centro y la comunidad de referencia. De igual modo, las experiencias de Aprendizaje-Servicio se organizan más como actuaciones puntuales, carentes de carácter transversal en el currículum.

- ¿Hasta qué punto promueven la participación? El carácter intervencionista de algunas experiencias, como el Aprendizaje-Servicio, implica ciertas limitaciones. Se parte de un diagnóstico a partir del cual se diseña un plan de actuación que trata de dar respuestas a necesidades "detectadas" (no necesariamente "sentidas" o "compartidas"). Son acciones que sin duda promueven servicios, pero no necesariamente asumen un enfoque participativo orientado a la transformación social y a cambios sistémicos (Westheirmer y Kahne, 2003). Este mismo carácter intervencionista se puede ver en los planteamientos que articulan las Ciudades Educadoras, que tratan de contribuir a una formación que permita crecer y hacer uso de las ciudades, supuestamente de la manera que la "ciudad" establece pero... ¿quién o quiénes deciden sobre tal uso?

- ¿Hablamos de participación o de representación? Algunas experiencias, como los Consejos Infantiles, proponen la formación de un grupo reducido para constituir estos órganos municipales.

- ¿En qué se concreta el contenido de algunas de estas experiencias? Supuestamente las autoras de este artículo vivimos en Ciudades Educadoras, sin que el día a día nos permita honestamente identificarlas como tales. La Ciudad Educadora se convierte así en un título carente de significado, sin que podamos encontrar en ella señales de apertura que permitan la gestión participativa de quienes viven en ella.

Por tanto, en nuestra opinión, estas propuestas se encuentran en un espacio de cierta ambigüedad. De ahí que sea necesario explorar experiencias de aprendizaje cercanas a una ciudadanía crítica y participativa, orientada a la transformación social.

\section{El DisCURSO DE LOS ACTORES}

Una línea emergente en la investigación sobre ciudadanía se centra en el análisis de los discursos y procesos a través de los que esta se define y se pone en juego, con el fin de comprender cómo se están construyendo sus significados y prácticas (Biesta, Lawy y Kelly, 2009; Hausendorf y Bora, 2006; Fairclough, Pardoe y Szerszynski, 2006; Knight-Abowitz y Harnish, 2006). En esta línea, nuestro proyecto se propone explorar los discursos y las experiencias de aprendizaje y práctica de la ciudadanía, con el fin de contribuir a su comprensión y a la 
formulación de propuestas educativas para su construcción desde un enfoque intercultural.

\subsection{Método}

Partiendo de tres interrogantes generales, qué es ciudadanía, cómo se ejerce y cómo se aprende, en una primera fase del proyecto hemos realizado un estudio exploratorio, que ha consistido en el desarrollo de 14 entrevistas en profundidad. Las entrevistas pretenden analizar significados asociados a la ciudadanía, formas de ejercerla, motivaciones y principios que la orientan, formas y espacios de aprendizaje e implicaciones educativas.

Para orientar el desarrollo de las entrevistas propusimos una serie de cuestiones generadoras que fueron utilizadas de manera flexible por los entrevistadores, tratando de favorecer la particularidad de los discursos y experiencias. Los ejes temáticos de la entrevista y la sugerencia de cuestiones concretas aparecen recogidos en el Cuadro 1.

Un aspecto esencial en el proceso ha sido la selección de los/as informantes. En este sentido, nos planteamos como criterio de selección el que las personas a entrevistar tuvieran una trayectoria de implicación social y participación comunitaria coherente con la idea de ciudadanía como construcción colectiva para la mejora de las condiciones de vida de los grupos. Asimismo, nuestro interés en indagar en el aprendizaje de la ciudadanía, no solo desde la óptica de la educación formal, sino también como un proceso de aprendizaje a lo largo de la vida, nos llevó a seleccionar actores cuya experiencia se desarrollara en distintos ámbitos de intervención:

- Educativo, referido tanto a la educación reglada como a la educación no formal.

- Movimientos sociales que inciden en la construcción y desarrollo de la ciudadanía.

- Teórico-académico, que incluye profesionales cuya investigación o/y docencia se desarrolla sobre este tema.

- Político, incluyendo la militancia en partidos, sindicatos u otras agrupaciones.

- Medios, con personas que trabajan en la información y comunicación en general.

De esta forma, los discursos a analizar ofrecen mayor variedad de experiencias y perspectivas en la construcción del significado y en el aprendizaje de la ciudadanía.

Una vez transcritas las entrevistas, se procedió al análisis de contenido. La Figura 1 presenta un mapa relacional con las principales categorías manejadas en el análisis global. 
1. Qué es, qué significa ser ciudadana o ciudadano

- Autopercepción como ciudadanos/as (ciudadanía formal-activa, individualcolectiva...).

- Quiénes son los ciudadanos/as: mujeres, niños y jóvenes, ancianos, minorías...

- Cuáles son los ámbitos de la ciudadanía (comunidad, ciudad, nación, mundo...).

- Ciudadanía y diferencia: qué parte del mundo constituye "lo común", cuál es el ámbito de la esfera pública, qué diferencias culturales deben considerarse en ella, qué cuestiones deben ser objeto de decisión colectiva.

2. Cómo se ejerce la ciudadanía y qué actividades implica

- $\quad$ En qué comunidades -grupos, organizaciones, movimientos, instituciones...se involucran nuestros informantes.

- Cuáles son sus finalidades, sus objetivos, de qué manera contribuyen a la transformación social, cómo conciben esta transformación.

- Cómo son esas comunidades, qué características tienen (cerradas-abiertas, flexibles-rígidas, permanentes-temporales...), en qué tipo de vinculación se basan (intereses, identidades, creencias...).

- Qué actividades desarrollan en ellas, qué tipo de procesos internos y externos ponen en marcha.

- Qué carácter democrático tienen, qué espacios de participación facilitan, cómo se desarrolla esa participación (quiénes participan, para qué, en qué grado).

- Límites y barreras al ejercicio de la ciudadanía (del propio sujeto, del entorno, del sistema...).

3. En qué principios, valores y motivaciones se basa su ejercicio

- Qué razones mueven a actuar, a implicarse.

- Hacia dónde, qué proyecto de sociedad proponen.

- Qué "valores" y cómo se conciben.

4. De qué formas se aprende: recursos, medios, habilidades que se ponen en juego

- Experiencias concretas, aprendizajes.

- Motivaciones.

- Modelos, referentes (personales, políticos, culturales...). 


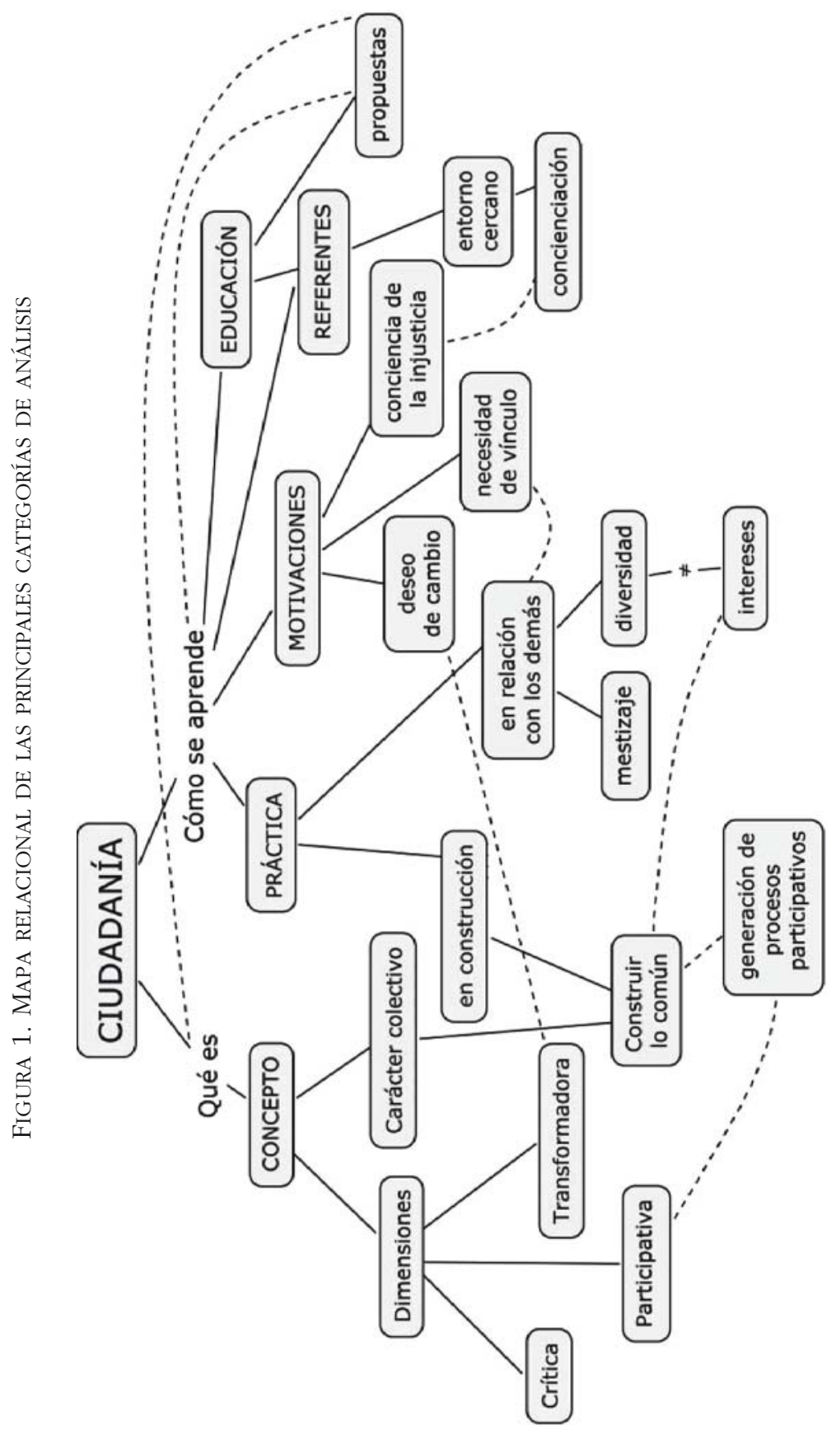


Los resultados que presentamos a continuación se centran en los aspectos relacionados con el aprendizaje y sus implicaciones educativas: ¿cómo se aprende la ciudadanía?, ¿qué motivaciones y referentes facilitan su aprendizaje y práctica?, ¿qué propuestas educativas podrían derivarse?

\subsection{Resultados}

Una de las primeras ideas que emergen del análisis es que la ciudadanía crítica y participativa se aprende. Este aprendizaje es un proceso de construcción permanente que se realiza más por la vía informal que por la formal. Es decir, se aprende a ser ciudadano/a "Siéndolo", poniendo en práctica la ciudadanía, participando en el mundo que nos rodea, implicándonos personalmente en los grupos, en los procesos colectivos y en la toma de decisiones.

Uno yo creo que aprende a amar amando, y aprende a ser ciudadano siendo ciudadano; yo creo que no es una cuestión de que cumplas 18 años, y puedas ejercer tu derecho al voto, que muchas veces es el ideal ¿no? (E1).

Muchas de las personas entrevistadas se refieren a su entorno familiar, especialmente madre o padre, como los primeros referentes en el aprendizaje de la ciudadanía. Se trata de familias preocupadas por los demás, que se involucran en los problemas sociales, que participan en su entorno para mejorarlo. Esta idea incide en la importancia que tiene la educación informal en la construcción de la ciudadanía. Así, las experiencias vividas en el entorno cercano constituyen una forma inicial de ejercer derechos y obligaciones que contribuye a la generación de una conciencia crítica y una actitud de cuestionamiento.

Tengo la suerte de que en mi familia siempre se ha dado un espíritu muy crítico mis padres... mi madre... siempre ha tenido mucho compromiso social, entonces yo eso ya lo llevaba de casa (E8).

Algunos profesores, compañeros/as del colegio o de la universidad y amigos son mencionados también en diversas ocasiones como referentes. De alguna forma, las personas que son significativas para cada uno tienen un papel fundamental en el desarrollo de quiénes somos, en línea con la concepción interaccionista de Mead (1973).

Se pone de manifiesto igualmente la importancia de las motivaciones en este proceso de aprendizaje. Se mencionan tres grandes razones para actuar: la necesidad de vínculos recíprocos (ser reconocido, sentirse parte, unirse a otros), la conciencia de la injusticia y el deseo de cambio, de transformación, de construcción de una sociedad mejor:

Esta idea de luchar por cambiar las cosas y que no haya tantas diferencias sociales entre la gente, y económicas, tanta situación de injusticia social, que es lo que siempre me ha movido (E2). 
Lo más importante [...] es lo que quieres construir en este mundo ¿no? Para mí eso es lo que me mueve (E10).

Un aspecto clave del aprendizaje de la ciudadanía es que no es un proceso en solitario; aprender ciudadanía implica necesariamente la relación con los demás, la construcción de colectividades con una finalidad.

La única manera en la que yo he podido sentirme ciudadano es cuando he podido, junto con otras personas, organizar espacios donde siento que participo y busco que juntos podamos participar más; y, desde luego, apropiarse de los espacios para la participación ciudadana con... con todas las dificultades que eso implica (E13).

En estos procesos, el grupo supone algo más que la suma de sus miembros; permite a los individuos crecer y aprender juntos, aportando el acompañamiento que refuerza los propios planteamientos. De ahí la necesidad de buscar aliados, de crear vínculos que permitan la consecución de fines comunes.

A lo mejor una obra de teatro es mucho mejor de profesionales, seguro que de amateur, pero la ventaja de que lo hagan amateur es que han estado tres meses juntándose siete personas y eso crea vínculos; y esos vínculos además se construyen y cuando haya un problema [...] esos siete tendrán vinculación para poder articular una protesta, una queja, o buscar una solución (E7).

En un trabajo similar realizado con docentes (Olmos, Carrillo, Martínez y Gaytán, 2011), las participantes en el estudio reconocían también la importancia de la colaboración para crear una comunidad de aprendizaje orientada al desarrollo de la ciudadanía.

Se manifiesta repetidamente en las entrevistas la necesidad de un mestizaje, en el sentido de que las personas se mezclen con otras diferentes que aporten puntos de vista distintos:

Me siento liberado de haberme quitado de en medio esos ambientes de totalizador [...] cuando lo hablo con otra gente, que nos dejábamos la piel por ideas y por las diferencias... y al final dices no, no, si tenemos que convivir juntos hay que aceptar gente que no piensa como tú (E12).

Es en la diversidad de ideologías, procedencias y opiniones donde se generan verdaderos procesos participativos. La diversidad implica estar abiertos a otras experiencias, aceptar otros puntos de vista y acoger nuevas ideas. Una apertura que requiere la aceptación del conflicto como parte del proceso y como una oportunidad para el aprendizaje y el crecimiento colectivo.

Pero tenemos como que a veces inseguridad en cuál sea la manera de hacerlo que no nos haga caer, finalmente, en formar... digamos, una secta o algo que se vea como muy cerrado porque no es... digamos, el espíritu de un movimiento abierto solicitando que cada vez más gente quiera participar y que no se tengan que afiliar a nada ino? (E13). 
CIUDADANÍA PARTICIPATIVA Y TRANSFORMADORA: ANÁLISIS DE DISCURSOS Y PROPUESTAS DE APRENDIZAJE

Si la ciudadanía se aprende, es posible educar en ciudadanía. Sin embargo, la asociación entre "educación" y "ciudadanía" conduce el discurso de forma espontánea hacia el debate actual en torno a la "educación para la ciudadanía" como asignatura impartida en la escuela. Se considera esta materia como un ingredientemás añadido al ingente repertorio de contenidos curriculares, y se cuestiona que pueda calar en un cambio educativo que ponga en práctica los principios de crítica, de creatividad, de transformación y de participación a todos los niveles:

Hombre, desde luego (aprender) a ser ciudadanos con una asignatura, quien se piense eso... entonces no... habría que ver ahí cómo... pero a mí la idea en sí de educar, me parece que es estupenda (E10).

Tratar de encasillar la educación para la ciudadanía en un pequeño espacio del horario escolar es negar la obviedad de que en cualquier situación estamos educando ciudadanos/as. Se cuestiona por ello el reduccionismo que supone tratar de poner límites a la educación de la ciudadanía, aunque se reconozca el papel que podría tener la escuela, considerando que la actual Ley de Educación la concibe como «el medio más adecuado para garantizar el ejercicio de la ciudadanía democrática, responsable, libre y crítica” (MEC, 2006a, Preámbulo).

Echo de menos que eso se haga a veces en el colegio, el que si hay una pelea entre dos niños [...] yo lo siento mucho pero las matemáticas y la lengua pasan a un segundo plano [...] con lo cual pues se para eso y hablamos de qué es lo que ha pasado y hablamos de lo que hay que hacer y por qué hay que hacerlo así, y entre todos vamos razonando cómo se llega a eso. Eso para mí sería una buena escuela de ciudadanos, sin necesidad de que haya de nueve y cuarto a diez y media educación para la ciudadanía, que me va a dar igual (E10).

Las personas entrevistadas, a través de sus experiencias y sus críticas a la escuela actual, ofrecen propuestas y señalan los cambios necesarios para una educación en ciudadanía. Una de las ideas que se destaca en este sentido es la necesidad de promover la participación y el diálogo como base de la práctica educativa:

La participación para mí es obligatoria [...] Priorizo las formas de comenzar a hablar y de obligar a hablar... forzando a que de alguna manera la participación sea el hilo conductor de la clase (E4).

Otros estudios (Olmos et al., 2011) también ponen de manifiesto la relevancia de la participación en el aprendizaje, defendiendo la necesidad de propiciar la implicación del alumnado no solo en las actividades de aula sino también en la comunidad circundante. Se trata de dar voz a los/as estudiantes para que puedan ejercer la ciudadanía, facilitándoles un espacio para poder tomar decisiones. Se incide así en la identificación entre aprendizaje y práctica.

Enfoques como las comunidades de aprendizaje se señalan como facilitadores de una educación democrática (Flecha, 2009), entendida como proyecto de escuela que se construye a través de la participación de todos. Se reivindica, por otra 
parte, una educación que promueva el diálogo entre todos los miembros de la comunidad educativa:

Ahora yo creo que sería mucho más eficaz de cara al docente que construya su legitimidad en base a su capacidad para dialogar (E2).

Asimismo se ve necesario desarrollar en nuestros/as estudiantes la capacidad de análisis y crítica que les permita cuestionar las razones que legitiman desigualdades sociales en el momento actual, heredadas como argumentos que se han mantenido a lo largo del tiempo y que privilegian a ciertos grupos frente a otros:

La desigualdad en nuestra sociedad, [...] pues ir transformando un poco eso y como que nuestras intervenciones van por que se transformen desde la base, [...] que lo cuestionen y que lo transformen y de algún modo que tengan libertad para elegir (E5).

Por otro lado, las personas entrevistadas proponen una educación que promueva la inquietud por la información. Saber qué ocurre a nuestro alrededor se plantea como parte del ejercicio de nuestra responsabilidad social. Es a partir de la información plural, contrastada y debatida, como podemos ejercer nuestra capacidad de análisis y crítica. Se acusa claramente una pérdida progresiva de este interés por conocer qué está pasando. No se juzga ni siquiera la credibilidad de los medios, como posible escudo que pretendiera justificar el desinterés; es un problema de apatía hacia lo que no nos afecta de forma directa o cercana:

¿Las claves? Información y herramientas para que la gente se informe, y crear inquietudes en la gente por conocer, el problema es que la sociedad actual está dormida, yo creo que en la sociedad actual parece que hay un gusto por la ignorancia, es como si dijeras no solo no sé nada sino que es que además me siento orgulloso de no saber porque es que no me importa (E8).

Además, se apuesta por una educación creativa, capaz de proponer alternativas transformadoras. Sorprenden las aportaciones de los niños/as recién escolarizados en actividades que tienen que ver con el desarrollo comunitario.

Aprovechando los presupuestos participativos [...] niños de tres años habían ahorrado dinero en el presupuesto que había en la escuela, porque daban otras ofertas, niños de tres años, niños de seis se habían transformado en personas creativas, donde en un momento determinado solo había receptividad (E14).

La educación para la transformación social exige el conocimiento de nuestros derechos y la toma de conciencia de nuestra propia capacidad de intervención sobre la realidad. En este sentido, para un abordaje completo de lo que significa educar en ciudadanía se hace necesario que la intervención escolar trascienda las paredes del aula y promueva espacios en los que se ponga en relación lo que ocurre en su interior y las repercusiones que para la vida de las personas y los 
pueblos tiene el diseño de los sistemas sociales, políticos y económicos en los que estamos inmersos (Caballero, 2002).

Se señala que la educación debe centrarse en las personas, antes que en los contenidos, lo que implica conceder importancia a las relaciones sociales. Este aspecto, teóricamente motor de la educación, está en la práctica relegado a un plano secundario debido al peso que ocupan las materias curriculares. La educación debe promover valores que sustenten la práctica de la ciudadanía, ofreciendo los referentes necesarios y permitiendo aprender sin autoritarismo el uso de determinadas "reglas" sociales:

El formar a la gente como persona, más que el formar a la gente como... como estudiante... que tiene que alcanzar una meta para prosperar en la vida. Yo creo que era un poco como darse cuenta de la diferencia entre lo importante y lo obligatorio, entonces lo obligatorio quedaba en un segundo plano porque estaba por delante lo importante... (E9).

\section{DisCUSIÓN Y CONCLUSIONES}

El análisis de las entrevistas nos ha permitido dibujar un mapa de discursos en torno a la ciudadanía en el que destaca la identificación entre aprendizaje y práctica. Se aprende a ser ciudadanos simplemente "siéndolo", o, lo que es lo mismo, participando en procesos basados en el diálogo, la construcción de vínculos con los otros, el mestizaje de ideologías, todo lo cual contribuye a la generación de una conciencia crítica. De forma semejante se expresan García Pérez y De Alba (2012, 304), quienes afirman que "a participar solo se aprende participando".

Ya que se aprende a ser ciudadanos "siéndolo", la familia se muestra como el primer agente educativo para una gran parte de nuestros informantes. Así, pertenecer a una familia con inquietud social y preocupación por el bien común ha sido un elemento clave en la configuración de la forma de ser y estar en el mundo de nuestros informantes. Otros referentes importantes son algunos profesores y compañeros que han contribuido al desarrollo de una conciencia y una actitud crítica ante lo que les rodea. Dado que los informantes han construido la ciudadanía a través de vías informales de aprendizaje basadas en la relación con los demás y la participación en grupos, es fácil entender su postura crítica a la idea de una educación ciudadana reducida a los estrechos límites de una materia curricular. La educación para la ciudadanía, más que ser transmitida, necesita ser practicada en la escuela (Borghi, 2012). ¿Cómo se puede aprender a ser ciudadanos a través de unos ejercicios de clase los lunes de doce a una? Es, sin duda, un contrasentido, lo cual no exime a la escuela de su responsabilidad de educar en ciudadanía. Sin embargo, esta misión solo puede realizarse si se ponen en práctica algunos principios básicos, que están en la base de la construcción de una ciudadanía participativa y crítica: 
- La escuela debe facilitar espacios en los que los estudiantes puedan poner en práctica la participación. Esto no necesariamente implica iniciar nuevos proyectos, pues la idea es que el estudiante participe en las actividades cotidianas, implicándose y tomando conciencia de la responsabilidad colectiva en todo lo que sucede a su alrededor. La participación y el diálogo han de ser la base de la enseñanza. Una participación que trascienda las paredes del aula y que facilite los espacios de poder necesarios para que los y las estudiantes puedan tomar decisiones.

- Dada la importancia que los referentes cercanos tienen en el desarrollo de la ciudadanía, y sabiendo que no todas las personas encuentran estos referentes en su familia, se debe apostar por un modelo de aprendizaje situado, que ofrezca la posibilidad de identificarse con "referentes de acción", personas del entorno con capacidad para desarrollar proyectos como miembros de una comunidad.

- La escuela debe aportar modelos diversos y enseñar desde la pluralidad de visiones, dejando a la persona en libertad para integrar lo recibido/ vivenciado en su propia identidad. La diversidad (de opiniones, de capacidades, de formas de ser) es positiva, rica, y es necesario reconocerla, valorarla y convivir en ella.

- Es necesaria una enseñanza que fomente la inquietud por la información combinada con el desarrollo de una actitud crítica y creativa, capaz de imaginar, compartir y construir alternativas necesarias para la transformación social.

- La organización escolar debe avanzar hacia la ruptura de jerarquías y funcionar desde un liderazgo entendido como coordinación y dinamización.

- Y, finalmente, la educación debe centrarse en las personas, antes que en los contenidos, lo que implica conceder importancia a las relaciones sociales y cultivar la sensibilidad y la empatía, considerando la dimensión afectiva necesaria en la comunicación.

Lo que vendría a corroborar la idea de que es más eficaz la educación por inmersión que la educación por transmisión (Tonucci, 2009). Estos principios, por otra parte, tendrían que extenderse a la formación de docentes, que debería alejarse de un enfoque academicista para potenciar la construcción de conocimiento de una forma colectiva y crítica. El problema es que en muchos casos el profesorado no tiene experiencia directa de participación ciudadana, lo que dificulta la asunción de un rol social comprometido con el aprendizaje de una ciudadanía activa (Schugurensky y Myers, 2003).

En esta fase de la investigación hemos seleccionado a los informantes en función de su trayectoria previa de implicación social y su compromiso con la idea de ciudadanía como construcción colectiva para la mejora de la realidad social. Apuntamos como una cierta limitación del estudio el hecho de que los resultados estén condicionados por sus ideologías y puntos de vista. Por otra 
parte, su enfoque basado exclusivamente en la entrevista no permite acceder a situaciones de discusión en las que el discurso se construye socialmente con el aporte recíproco de otras personas, como sucede en los grupos de discusión.

Por ello las fases siguientes de esta investigación vinculan decires y haceres desde otras perspectivas. Aunque consideramos el discurso como una forma de práctica, encontramos conveniente profundizar en los objetivos planteados a través de estudios de caso múltiples en los que podamos combinar otras técnicas de recogida de información, como observaciones e historias de vida. Asimismo, considerando la dimensión participativa de la ciudadanía, nos proponemos impulsar procesos de construcción conjunta de significados y prácticas colectivas. Para ello, se han planificado diferentes acciones y tareas que pretenden entretejer una red de investigación e intervención para la construcción de una ciudadanía crítica, participativa y transformadora.

\section{REFERENCIAS BIBLIOGRÁFICAS}

ABDallah-Pretceille, M. (2001) La Educación Intercultural. Barcelona, Idea.

Aguado, T. (coord.) (2006) Educación Intercultural: necesidades de formación de profesorado desde la perspectiva europea/Intercultural Education: Teacher training needs from a European perspective. Madrid, UNED.

- (2010) Escuelas para todos, en Aguado, T. (coord.) Diversidad e Igualdad en Educación. Madrid, UNED, 163-189.

Argibay, M.; Celorio, G.; Celorio, J. J. y López de Munain, A. (2011) Educación para la Ciudadanía: Informe sobre la situación en ocho comunidades autónomas. VitoriaGasteiz, Hegoa.

Bargalló, A. (2008) De la utopía a la práctica. Cuadernos de Pedagogía, 375, 57-60.

Benedicto, J. y OráN, M. L. (2003) Los jóvenes, ¿̨ciudadanos en proyecto?, en Benedicto, J. y Morán, M. L. Aprendiendo a ser ciudadanos. Experiencias sociales y construcción de la ciudadanía entre los jóvenes. Madrid, INJUVE, 39-64.

Biesta, G.; LAWY, R. y Kelly, N. (2009) Understanding young people's citizenship learning in everyday life: The role of contexts, relationships and dispositions. Education, Citizenship and Social Justice, 4 (1), 5-24.

Borghi, B. (2012) Yo, ciudadano en el ciento cincuenta aniversario de la unificación de Italia. La educación para la ciudadanía, una inversión para el futuro, en DE ALBA, N.; García Pérez, F. F. y SAntiesteban, A. (eds.) Educar para la participación ciudadana en la enseñanza de las ciencias sociales, 1. Sevilla, Asociación Universitaria de Profesorado de Didáctica de las Ciencias Sociales, 317-330.

CABallero, A. (2002) Educación para la paz y la convivencia: implicaciones para la gestión de conflictos escolares. Cultura y Educación, 14 (4), 403-411.

Capella, J. R. (2005) Los ciudadanos siervos. Madrid, Editorial Trotta.

Da Silveira, P. (2009) Educación cívica: tres paradigmas alternativos, en Rubio CarRaCEdo, J.; Rosales, J. M. y Toscano, M. (dirs.) Democracia, ciudadanía y educación. Madrid, Akal/Universidad Internacional de Andalucía, 201-220.

Dejaeghere, J. G. (2009) Educación ciudadana crítica para una sociedad multicultural. Revista Interamericana de Educación para la Democracia, 2 (2), 225-240. Consultado 
el 20 de abril de 2012. http://scholarworks.iu.edu/journals/index.php/ried/article/ viewFile/159/284.

Dietz, G. (2003) Multiculturalismo, interculturalidad y educación: una aproximación antropológica. Granada, Universidad.

Fairclough, N.; PARdoe, S. y Szerszynski, B. (2006) Critical discourse analysis and citizenship, en Hausendorf, H. y Bora, A. (eds.) Analysing Citizenship Talk. Amsterdam/ Philadelphia, John Benjamins Publishing Company, 98-123.

FleCHA, R. (2009) Cambio, inclusión y calidad en las comunidades de aprendizaje. Cultura y Educación, 21 (2), 157-169.

FolgueIRAS, P. (2005) De la tolerancia al reconocimiento: programa de formación para una ciudadanía intercultural. Tesis Doctoral, Facultad de Pedagogía, Universidad de Barcelona. Consultada el 12 de marzo de 2012. http://hdl.handle.net/10803/2351.

García Pérez, F. F. y De Alba Fernández, N. (2012) La educación para la participación ciudadana entre dos polos: el simulacro escolar y el compromiso social, en DE ALBA, N.; García Pérez, F. F. y SAntiesteban, A. (eds.) Educar para la participación ciudadana en la enseñanza de las ciencias sociales, 1. Sevilla, Asociación Universitaria de Profesorado de Didáctica de las Ciencias Sociales, 297-306.

HAusendorf, H. y BORA, A. (2006) Communicating citizenship and social positioning, en Hausendorf, H. y Bora, A. (eds.) Analysing Citizenship Talk. Amsterdam/Philadelphia, John Benjamins Publishing Company, 23-49.

HeAter, D. (2007) Ciudadanía. Una breve historia. Madrid, Alianza Editorial.

IKEDA, S. (2004) Imperial subjects, national citizenship and corporate subjects: cycles of political participation/exclusion in the modern world-system. Citizenship Studies, 8 (4), 333-347.

KNIGHT-ABOwITZ, K. y Harnish, J. (2006) Contemporary discourses of citizenship. Review of Educational Research, 76 (4), 653-690.

López Ruiz, J. I. (2008) Construyendo escuelas democráticas. Barcelona, Hipatia.

MALIK, B. y MATA, P. (2009) Identifying training needs in relation to diversity and intercultural education: the case of Spain, en Czerka, E. y MechlinsKa-PAuli, M. (eds.) Teaching and Learning in different cultures. An Adult Education perspective. Gdansk, Higher School of Humanities Press, 34-45.

Marshall, T. H. (1998) Ciudadanía y clase social. Madrid, Alianza.

MARTINELLI, S. (coord.) (2000) Intercultural learning T-kit. Estrasburgo, Council of Europe/ European Commission. Consultado el 10 de febrero de 2012. http://www.youth-partnership.net/export/sites/default/youth-partnership/documents/Publications/T_kits/4/ content.pdf.

Mata, P. (2010) Ciudadanía y participación democrática, en Aguado, T. (coord.) Diversidad e igualdad en educación. Madrid, UNED, 125-156.

Mata, P. y Ballesteros, B. (2012) Diversidad cultural, eficacia escolar y mejora de la escuela: encuentros y desencuentros. Revista de Educación, 358, 17-37.

Mead, H. (1973) Espiritu, persona y sociedad. Barcelona, Paidós [V. O. Mind, Self and Society. Londres, The University of Chicago Press, 1934].

MEC (2006a) Ley Orgánica 2/2006, de 3 de mayo, de Educación. Consultado el 4 de marzo de 2012. http://www.boe.es/boe/dias/2006/05/04/pdfs/A17158-17207.pdf.

- (2006b) Real Decreto 1631/2006, de 29 de diciembre por el que se establecen las enseñanzas minimas correspondientes a la Educación Secundaria Obligatoria. Consultado el 4 de marzo de 2012. http://www.boe.es/boe/dias/2007/01/05/pdfs/A00677-00773.pdf. 
- (2012) Texto borrador del anteproyecto de Ley Orgánica para la mejora de la calidad educativa. Consultado el 20 de enero de 2013. http://www.mecd.gob.es/dms/mecd/ ministerio-mecd/servicios-al-ciudadano/participacion-publica/lomce/20121219-borrador-lomce.pdf.

Molina, F. (2000) Apuntes para una sociología de la educación intercultural. Cultura y Educación, 12 (1-2), 167-179.

Moro, G. (2008) La evaluación de los ciudadanos de la participación pública, en Evaluación de la participación pública en la elaboración de políticas públicas. Madrid, Instituto Nacional de Administración Pública/OCDE, 121-140.

MougÁn, J. C. (2009) Hacia una teoría de la educación para una ciudadanía democrática, en Rubio Carracedo, J.; Rosales, J. M. y Toscano, M. (dirs.) Democracia, ciudadanía y educación. Madrid, Akal/Universidad Internacional de Andalucía, 221-251.

Olmos, A.; Carrillo, A.; Martínez, R. M. y GaYtán, M. (2011) Los sentidos construidos por el docente sobre su práctica en la educación ciudadana. El empoderamiento en la escuela secundaria. Cultura y Educación, 23 (4), 559-57.

Puig Rovira, J. M.; Gijón Casares, M.; Martín García, X. y Rubio Serrano, L. (2011) Aprendizaje-servicio y educación para la ciudadanía. Revista de Educación, n.. extraordinario 2011, 45-67.

RAmírez, J. L. (1995) La ciudad y el sentido del quehacer ciudadano. Lleida, Universidad.

Rius, M. (2010) El ciudadano sin atributos, en CAmps, V. (ed.) Democracia sin ciudadanos. La construcción de la ciudadanía en las democracias liberales. Madrid, Editorial Trotta, 17-36.

Rubio Carracedo, J. (2003) Educar ciudadanos: el planteamiento republicano-liberal de Rousseau. Contrastes, Revista Internacional de Filosofia, suplemento 8, 211-227.

SCHugurensky, D. (2005) Citizenship and citizenship education. Consultado el 3 de mayo de 2012. http://fcis.oise.utoronto.ca/ daniel_schugurensky/lclp/c\&ce.html.

SCHugurensky, D. y MYers, J. (2003) Learning to teach citizenship: A lifelong learning approach. Encounters on Education, 4, 145-166.

- (2008) Informal learning through engagement with local democracy: The case of the Seniors' Task Force of Healthy City Toronto, en CHURCH, K.; BASCIA, N. y SHRAGGe, E. (eds.) Learning through Community: Exploring Participatory Practices. Springer, 73-96. Consultado el 28 de abril de 2012. http://www.oise.utoronto.ca/legacy/research/ edu20/documents/seniorstaskforce.pdf.

SEOANE, J. (2003) Del moralismo que necesitamos y algunas de sus incomodidades. Contrastes, Revista Internacional de Filosofía, suplemento 8, 229-244.

SIIM, B. (2000) Gender and citizenship: Politics and agency in France, Britain and Denmark. Cambridge, University Press.

Susín, R. (2008) Ciudadanía, en García Inda, A. y Marcuello, C. (coords.) Conceptos para pensar el siglo XXI. Madrid, Catarata, 25-54.

TonuCCI, F. (2009) ¿Se puede enseñar la participación? ¿Se puede enseñar la democracia? Investigación en la Escuela, 68, 11-24.

Trilla Bernet, J. y Novella Cámara, A. M. (2011) Participación, democracia y formación para la ciudadanía. Los consejos de infancia. Revista de Educación, n. ${ }^{\circ}$ extr. 2011, 23-43.

Turner, B. S. (1990) Outline of a theory of citizenship. Sociology, 24, 189-217.

- (1993) Citizenship and social theory. Londres: Sage.

- (2001) The erosion of citizenship. The British Journal of Sociology, 52 (2), 189-209.

Westheimer, J. y Kahne, J. (2003) What kind of citizen? Political choices and educational goals. Encounters on Education, vol. 4, 47-64. 